PROCEEDINGS OF THE AMERICAN MATHEMATICAL SOCIETY

Volume 124, Number 9, September 1996

\title{
REMARKS ON THE LOCAL HOPF'S LEMMA
}

\author{
VLADIMIR SHKLOVER
}

(Communicated by J. Marshall Ash)

\begin{abstract}
The paper deals with the problem of extending the recent work of M.S.Baouendi and L.P.Rothschild concerning harmonic functions vanishing to infinite order in the normal direction in balls and half-spaces. Contrary to what one expects, we show that the B.-R. result extends neither to arbitrary domains nor to cases when the normal is replaced by a curve transversal to the boundary. The exact criterion when the result holds in $\mathbf{R}^{2}$ is given.
\end{abstract}

\section{INTRODUCTION}

Consider a domain $D$ in $\mathbf{R}^{n}$ with a smooth boundary $\Gamma$. Let $\Omega$ be an open neighborhood of a point $x_{0}$ on $\Gamma$. Set $\Omega^{+}=\Omega \cap D, \Omega^{-}=\Omega \backslash \bar{D}, V=\Omega \cap \Gamma$. Assume that $\Omega^{+}, \Omega^{-}$are connected and $V$ is real analytic.

A continuous function $u$ in $\overline{\Omega^{+}}$is said to vanish to infinite order at $x_{0}$ if for every positive integer $N$ :

$$
\lim _{\substack{x \in \Omega^{+} \\ x \rightarrow x_{0}}} \frac{u(x)}{\left|x-x_{0}\right|^{N}}=0 .
$$

Also, $u$ vanishes to infinite order in a direction $\vec{b}$ at $x_{0}$, where $\vec{b}$ is a unit vector pointing inside $\Omega^{+}$and transversal to $\Gamma$, if for every $N$ :

$$
\lim _{t \rightarrow 0^{+}} \frac{u\left(x_{0}+t \vec{b}\right)}{t^{N}}=0 .
$$

Finally, $u$ vanishes to infinite order on a non-singular smooth arc $S: x=\gamma(t)$, $0 \leq t \leq 1$, in $\Omega^{+}$passing through $x_{0}$ and transversal to $\Gamma$ if for all $N$ :

$$
\lim _{t \rightarrow 0^{+}} \frac{u(x(t))}{t^{N}}=0 .
$$

(One can easily check that the definition (0.3) does not depend on the parametrization.)

M.S.Baouendi and L.P.Rothschild proved in [1] the following theorem.

(A). Let $D$ be either a ball or a half-space in $\mathbf{R}^{n}$. If $u$ is harmonic in $\Omega^{+}$and continuous in $\overline{\Omega^{+}}$, vanishes to infinite order in the normal direction at $x_{0}$, and $u(x) \geq 0$ for $x \in V$, then $u(x) \equiv 0$ in some neighborhood of $x_{0}$ in $V$.

This theorem implies the corollary

Received by the editors July 6, 1994 and, in revised form, February 28, 1995.

1991 Mathematics Subject Classification. Primary 30B40; Secondary 30C20, 30D50.

Key words and phrases. Harmonic functions, Hopf's lemma, analytic continuation.

(C)1996 American Mathematical Society 
(B). If, in addition to the hypotheses in (A), $u$ vanishes to infinite order at $x_{0}$, then $u(x) \equiv 0$ in $\Omega^{+}$.

Earlier, somewhat similar but weaker results under the hypothesis that $u$ is harmonic in a half-plane and decays exponentially along the $y$-axis were obtained in [4]. Also see further extensions by H.Shapiro [5], [6].

The authors in [1] remarked that similar results should, perhaps, hold in arbitrary domains $D$ (in fact, only a small real-analytic piece $V$ of the boundary $\Gamma$ matters). An interesting question is also whether one can replace the normal direction by an oblique one, or even by a real-analytic or $C^{\infty}$ curve transversal to $\Gamma$. More precisely, does the following generalization of $(\mathrm{A})$ hold?

$\left(\mathbf{A}^{\prime}\right)$. If $u$ is harmonic in $\Omega^{+}$and continuous in $\overline{\Omega^{+}}$, vanishes to infinite order in a direction $\vec{b}$ (or on an arc $S$ ) at $x_{0}$, and $u(x) \geq 0$ for $x \in V$, then $u(x) \equiv 0$ in some neighborhood of $x_{0}$ in $V$.

Accordingly, a generalization of Corollary (B) is:

$\left(\mathbf{B}^{\prime}\right)$. If, in addition to the hypotheses in $\left(\mathrm{A}^{\prime}\right), u$ vanishes to infinite order at $x_{0}$, then $u(x) \equiv 0$ in $\Omega^{+}$.

It turns out that in general answers to the questions about $\left(\mathrm{A}^{\prime}\right)$ are in the negative. For general domains and transversal curves $\left(\mathrm{A}^{\prime}\right)$ does not hold. The subject of this paper is characterization of plane domains for which $\left(\mathrm{A}^{\prime}\right)$ is still true. Our main result (Theorem 2) is that $\left(\mathrm{A}^{\prime}\right)$ holds if and only if $V$ is locally symmetric about the normal to $\Gamma$ at $x_{0}$. However, $\left(\mathrm{B}^{\prime}\right)$ holds for all domains. We also include some results for the situation when the normal is replaced by a transversal curve.

\section{Examples of failure of $\left(\mathrm{A}^{\prime}\right)$}

As usual we identify $\mathbf{R}^{2}$ with $\mathbf{C}, x+i y=z \in \mathbf{C}, x, y \in \mathbf{R}$. Let $V$ contain $z=0$ and be defined by

$$
y=f(x),
$$

where $f(x)$ is a real-analytic function, $f(0)=0$ and the domain $D$ near the origin lies "above" $V$. Consider the above problem with $u(x, y)$ vanishing to infinite order on the $y$-axis $(y \geq 0)$ at $z=0 . f(x)$ has Taylor expansion near $x=0$

$$
f(x)=\sum_{k=1}^{\infty} c_{k} x^{k},
$$

where the coefficients $c_{k}$ are real. If $c_{1} \neq 0$, the $y$-axis is oblique with respect to $V$. If, in particular, all $c_{k}, k \geq 2$, vanish, $V$ is a piece of the straight line $y=c_{1} x$ and we have the half-plane situation with $u$ vanishing to infinite order in an oblique direction. If $c_{1}=f^{\prime}(0)=0$, i.e. $V$ is tangent to the $x$-axis at $z=0$, then the $y$-axis is normal to $V$. We can rewrite (1.2) as

$$
f(x)=\sum_{k=k_{0}}^{\infty} c_{k} x^{k},
$$

where $k_{0} \geq 1$, and $c_{k_{0}} \neq 0$ is the first non-vanishing coefficient in (1.2). The harmonic function

$$
u(x, y)=\operatorname{Im} \sqrt{1+z^{2}}
$$


is continuous in a sufficiently small neighborhood of the origin (we choose the branch of $\sqrt{z}$ so that $\left.\sqrt{1+0^{2}}=1\right)$. On the $y$-axis $z=i y$, so $u(x, y)=\operatorname{Im} \sqrt{1-y^{2}} \equiv 0$ (for $|y|<1$ ) and therefore $u(x, y)$ vanishes to infinite order on the $y$-axis at $z=0$. To check the hypotheses of $\left(\mathrm{A}^{\prime}\right)$ it remains to show that $u(x)$ has a constant sign on $V$.

Lemma 1.1. If $k_{0}=2 p+1$, where $p \geq 0$ is an integer and $c_{k_{0}}>0\left(\right.$ or $\left.c_{k_{0}}<0\right)$, then $u(x, y)>0(u(x, y)<0)$ in a neighborhood of 0 for $z \neq 0$ on $V$.

Proof. Without loss of generality suppose $c:=c_{k_{0}}>0$. From (1.3) we have:

$$
f(x)=c x^{2 p+1}+O\left(x^{2 p+2}\right) .
$$

Let us use the notation $O_{R}(\ldots)$ to say that $O(\ldots)$ is real-valued. Then for $z \in V$ we have $z=x+i\left(c x^{2 p+1}+O_{R}\left(x^{2 p+2}\right)\right)$ and

$$
\begin{aligned}
u(x, y) & =\operatorname{Im} \sqrt{1+\left\{x+i\left[c x^{2 p+1}+O_{R}\left(x^{2 p+2}\right)\right]\right\}^{2}} \\
& =\operatorname{Im} \sqrt{1+O_{R}\left(x^{2}\right)+2 i c x^{2 p+2}+O\left(x^{2 p+3}\right)} \\
& =\operatorname{Im}\left(1+O_{R}\left(x^{2}\right)+i c x^{2 p+2}+O\left(x^{2 p+3}\right)\right)=c x^{2 p+2}+O_{R}\left(x^{2 p+3}\right),
\end{aligned}
$$

so

$$
u(x, f(x))=c x^{2 p+2}+O_{R}\left(x^{2 p+3}\right) .
$$

Thus, for sufficiently small $|x| \neq 0, \quad u(x, f(x))>0$. Therefore, all the hypotheses of $\left(\mathrm{A}^{\prime}\right)$ are satisfied but $\left(\mathrm{A}^{\prime}\right)$ nevertheless fails $(u$ does not vanish anywhere on $V$ near 0 ). Thus, we have the following corollaries:

Corollary 1.2. $\left(\mathrm{A}^{\prime}\right)$ fails if one replaces the normal by an arbitrary line oblique with respect to $V$.

Corollary 1.3. $\left(\mathrm{A}^{\prime}\right)$ for an arbitrary domain $D$ fails for all $V$ as in $(1.5)$ provided that $p \geq 1\left(k_{0} \geq 3\right)$.

In general, $u(x, y)$ does not have to vanish not only on $V$ but also on the $y$-axis (i.e., on a normal or oblique line) and, moreover, does not have to extend across $V$ as a real-analytic function, contrary to what the Baouendi-Rothschild Theorem implies [1]. To see this, consider modified examples:

$$
u_{1}(x, y)=\operatorname{Im} \sqrt{1+z^{2}}+\operatorname{Im}\left(e^{-1 / \sqrt{z / i}}\right)
$$

(the branch cut for $\sqrt{z}$ is on the negative $y$-axis), so $u_{1}$ vanishes on the $y$-axis and is not analytic at $z=0$, or

$$
u_{2}(x, y)=\operatorname{Im} \sqrt{1+z^{2}}+\operatorname{Re}\left(e^{-1 / \sqrt{z / i}}\right),
$$

which does not vanish on the $y$-axis and is not analytic at $z=0$.

\section{Domains In Which $\left(\mathrm{A}^{\prime}\right)$ DOES HOLD}

Now consider $V$ defined as in (1.1) and such that the $y$-axis is orthogonal to $V$, i.e.

$$
y=f(x), \quad f(0)=f^{\prime}(0)=0 .
$$

$V$ is the image of a neighborhood of the origin on the $x$-axis under the map:

$$
\tilde{f}(z)=z+i f(z)=z+i c_{k_{0}} z^{k_{0}}+O\left(z^{k_{0}+1}\right) .
$$


Since $\tilde{f}^{\prime}(0)=1 \neq 0$, the inverse map $h(z)$ to $\tilde{f}(z)$ exists in a neighborhood of $z=0$ and

$$
h(z)=z-i c_{k_{0}} z^{k_{0}}+O\left(z^{k_{0}+1}\right) .
$$

Under this map a part of $V$ near the origin is mapped into the $x$-axis while the $y$-axis is mapped into a real-analytic arc $S$ defined by

$$
x=\varphi(y), \quad \varphi(0)=\varphi^{\prime}(0)=0 .
$$

Since harmonicity of the function $u(x, y)$ is preserved under conformal maps (together with the property of vanishing to infinite order), we have the following simple

Proposition 2.1. ( $\left.\mathrm{A}^{\prime}\right)$ holds for a boundary arc $V$ and a harmonic function $u(x, y)$ vanishing to infinite order on the $y$-axis at $z=0$ if and only if it holds for the $x$-axis and for $u(\tilde{f}(z))$ vanishing to infinite order on the arc $S$.

If $f(z)$ is an odd function, then $\tilde{f}(z)$ is odd and so is $h(z)$. Hence, $S$ must be symmetric about the origin in a small neighborhood of it, i.e. $\varphi(y)$ must also be an odd function:

$$
\varphi(y)=(-1)^{p} c y^{2 p+1}+O\left(y^{2 p+3}\right) .
$$

In a more general case, when $f(x)$ is as in (1.5) (with the same notation),

$$
\varphi(y)=(-1)^{p} c y^{2 p+1}+O\left(y^{2 p+2}\right) .
$$

Conversely, given the arc $S$ (by (2.4)) we can find the equation (2.1) of $V$ using a procedure similar to the above. Again, when $\varphi(y)$ is odd or given by (2.6), then $f(x)$ is odd or given by (1.5). From Proposition 2.1 and Corollary 1.3 we obtain the following

Corollary 2.2. Let a boundary arc $V$ be a part of the real axis. Let $\varphi(y)$ be the defining function (2.4) of an arc $S$ tangent to the imaginary axis. If the lowest non-zero term in the Taylor expansion of $\varphi$ is odd (2.6), then $\left(\mathrm{A}^{\prime}\right)$ always fails for harmonic functions $u(x, y)$ vanishing to infinite order on the arc $S$. In particular, $\left(\mathrm{A}^{\prime}\right)$ fails for arcs symmetric about the origin, i.e. for odd functions $\varphi$.

The following lemma is known in connection with Poincare's local problem of conformal geometry (cf. [2] and, also, [3, Lemma 1]). For the reader's convenience we supply a simple proof.

Lemma 2.3. Given an arc $V$ (or $S$ ), we can find a conformal map sending $V$ into the $x$-axis ( $S$ into the $y$-axis), $z=0$ to $z=0$ and the $y$-axis ( $x$-axis) into itself if and only if $V$ is locally symmetric about the $y$-axis ( $S$ is locally symmetric about the $x$-axis).

Proof. It suffices to prove the lemma for the $x$-axis and the arc $S$. Suppose $\varphi(y)$ is even, i.e., $\varphi(y)=\sum_{m=1}^{\infty} c_{2 m} y^{2 m}$ and the points of $S: \quad z=\varphi(y)+i y$ are the images of those on the $y$-axis under the map $\tilde{\varphi}(z)=z+\sum_{m=1}^{\infty}(-1)^{m} c_{2 m} z^{2 m}$. Since the coefficients of $\tilde{\varphi}(z)$ are real, the inverse map $h(z)$ to $\tilde{\varphi}(z)$ also has real coefficients. Thus, the $x$-axis is preserved under $h(z)$ while $S$ is mapped into the $y$-axis.

Conversely, suppose $h(z)$ is the required function. It preserves the real axis and so does its inverse $h^{-1}(z)$. Then $S$ is the image of the $y$-axis under the map $h^{-1}(z)$. 
By the Schwarz Reflection Principle $h^{-1}(\bar{z})=\overline{h^{-1}(z)}$ and $S$ is symmetric about the $x$-axis. The proof is complete.

Corollary 2.4. If a boundary $V$ given by (2.1) is symmetric with respect to the $y$-axis, i.e. the function $f(x)$ is even, then $\left(\mathrm{A}^{\prime}\right)$ holds.

Corollary 2.5. If a boundary arc $V$ is a part of the real axis and a function $u(x, y)$ harmonic in the domain $D(\partial D \supset V), u \geq 0$ on $V$, vanishes to infinite order on a part of an analytic arc $S$ orthogonal to the real axis while the whole curve $S$ is symmetric with respect to the $x$-axis, then u must vanish identically on some subinterval of $V$ about the origin, i.e. the conclusion of $\left(\mathrm{A}^{\prime}\right)$ still holds.

Note the following obvious corollary of Proposition 2.1:

Corollary 2.6. ( $\left.\mathrm{B}^{\prime}\right)$ holds for any real-analytic arc $V$.

Now we are ready to characterize configurations for which $\left(\mathrm{A}^{\prime}\right)$ holds. Without loss of generality, consider the problem when $V$ is a part of the $x$-axis and $u(x, y)$ vanishes to infinite order on an analytic arc $S$ tangent to the $y$-axis at the origin. $S$ is given by (2.4). If $\varphi(y)$ is even, i.e. its Taylor expansion near $y=0$ contains only even powers, then Corollary 2.5 implies that $\left(\mathrm{A}^{\prime}\right)$ holds. If the lowest non-zero term in the Taylor expansion of $\varphi$ is odd and $\varphi$ has the form (2.6), then $\left(\mathrm{A}^{\prime}\right)$ fails in view of Corollary 2.2. Thus, the only remaining case is when $\varphi(y)$ has the expansion

$$
\varphi(y)=c_{2} y^{2}+\ldots+c_{2 p} y^{2 p}+c_{2 p+1} y^{2 p+1}+O\left(y^{2 p+2}\right), \quad c_{2 p+1} \neq 0 .
$$

The curve $S$ is the image of the $y$-axis under the map

$$
g(z)=z-c_{2} z^{2}+\ldots+(-1)^{p} c_{2 p} z^{2 p}+i(-1)^{p+1} c_{2 p+1} z^{2 p+1}+O\left(z^{2 p+2}\right) .
$$

Set $h(z)=z-c_{2} z^{2}+\ldots+(-1)^{p} c_{2 p} z^{2 p}$, so that

$$
g(y i)=h(y i)+c_{2 p+1} y^{2 p+1}+O\left(y^{2 p+2}\right) .
$$

Now let $\zeta=h^{-1}(w)$ be the inverse of $h$ that has expansion

$$
h^{-1}(w)=w+d_{2} w^{2}+\ldots+d_{2 p+1} w^{2 p+1}+O\left(w^{2 p+2}\right),
$$

where all $d_{k}$ are real. Obviously, $h^{-1}$ sends the $x$-axis into itself (locally). The image of $S$ under $h^{-1}$ is a curve $\tilde{S}$. In view of (2.9) $\tilde{S}$ can be parametrized by

$$
h^{-1}(g(y i))=h^{-1}\left(h(y i)+c_{2 p+1} y^{2 p+1}+O\left(y^{2 p+2}\right)\right),
$$

and according to (2.10) we have:

$$
\begin{aligned}
h^{-1}(g(y i)) & =h^{-1}(h(y i))+c_{2 p+1} y^{2 p+1}+O\left(y^{2 p+2}\right) \\
& =y i+c_{2 p+1} y^{2 p+1}+O\left(y^{2 p+2}\right) \\
& =\left(y+O_{R}\left(y^{2 p+2}\right)\right) i+c_{2 p+1} y^{2 p+1}+O_{R}\left(y^{2 p+2}\right) .
\end{aligned}
$$

So in the $\zeta$-plane, $\tilde{S}$ is parametrized by

$$
\tilde{x}:=\operatorname{Re} \zeta=c_{2 p+1} y^{2 p+1}+O_{R}\left(y^{2 p+2}\right), \quad \tilde{y}:=\operatorname{Im} \zeta=y+O_{R}\left(y^{2 p+2}\right)
$$

or, excluding $y$, we obtain

$$
\tilde{x}=\tilde{\varphi}(\tilde{y})=c_{2 p+1} \tilde{y}^{2 p+1}+O\left(\tilde{y}^{2 p+2}\right),
$$

i.e. $\tilde{S}$ satisfies (2.6), and $\left(\mathrm{A}^{\prime}\right)$ fails because of Corollary 2.2. Applying Corollary 2.5 we now obtain 
Theorem 1. In the case when the arc $V$ lies on the real axis and $u(x, y)$ is vanishing to infinite order on an analytic arc $S$ tangent to the imaginary axis at the origin, $\left(\mathrm{A}^{\prime}\right)$ holds if and only if $S$ is locally symmetric about the $x$-axis, i.e. the defining function of $S(2.4)$ is even. In particular, if $S$ is a circular arc, $\left(\mathrm{A}^{\prime}\right)$ always holds.

Applying Proposition 2.1 and Lemma 2.3 we deduce

Theorem 2. In the case of an arbitrary domain $D$ with an arc $V$ on the boundary tangent to the real axis at the origin and a function $u(x, y)$ harmonic in $D$ and vanishing to infinite order on the imaginary axis, $\left(\mathrm{A}^{\prime}\right)$ holds if and only if $V$ is locally symmetric about the imaginary axis, i.e. its defining function (1.1) is even.

Finally, for the most general configuration of a boundary arc $V \subset \partial D$ and an analytic arc $S \subset D$ orthogonal to $V$ we have

Theorem 3. For a harmonic function $u(x, y)$ in $D$ vanishing to infinite order on the arc $S$ and non-negative on a boundary arc $V,\left(\mathrm{~A}^{\prime}\right)$ holds if and only if there is a conformal map that sends $V$ and $S$ respectively into the real and imaginary axes. In particular, if $S$ and $V$ are mutually orthogonal circular arcs, $\left(\mathrm{A}^{\prime}\right)$ holds.

Corollary 2.7 ("Conjugacy principle"). For the configuration of a boundary arc $V \subset \partial D$ and harmonic functions in $D$ vanishing to infinite order on an analytic arc $S$ orthogonal to $V,\left(\mathrm{~A}^{\prime}\right)$ holds if and only if it holds for the "dual configuration" of the domain $D^{\prime}: \partial D^{\prime} \supset S$ and harmonic functions in $D^{\prime}$ vanishing to infinite order on the arc $V$.

Also, note that if $S$ is a $C^{\infty}$-curve such that its defining function $\varphi(y)$ vanishes to infinite order at $y=0$, then $\left(\mathrm{A}^{\prime}\right)$ obviously holds for $V \subset \mathbf{R}$ and harmonic functions $u$ vanishing to infinite order on $S$ (the proof is similar to that in [1]).

\section{ACKNOWLEDGMENTS}

I wish to thank Dr. Dmitry Khavinson for drawing my attention to this problem and valuable advice. I am also grateful to Ms. Svetlana Levitan for her help in preparing this paper.

\section{REFERENCES}

[1] M.S.Baouendi and L.P.Rothschild, A local Hopf lemma and unique continuation for harmonic functions, Duke Math.J. 71, International Mathematics Research Notices 8 (1993), 245-251. MR 94i:31008

[2] P.J.Davis, The Schwarz Function and its Applications, The Carus Mathematical Monographs, MAA, 1974. MR 53:11031

[3] D.Khavinson, On reflection of harmonic functions in surfaces of revolution, Complex Variables 17 (1991), 7-14. MR 92j:31005

[4] B.F.Logan,Jr., Properties of high-pass signals, Dissertation presented to the Electrical Engineering Faculty of Columbia University, New York, 1965.

[5] H.S.Shapiro, Functions with a spectral gap, Bull.A.M.S. 79 (1973), 355-360. MR 49:7696

[6] H.S.Shapiro, Notes on a Theorem of Baouendi and Rothschild, TRITA-MAT-1994-0022, Royal Inst. of Tech., Stockholm, Sweden. CMP 95:17

Department of Mathematical Sciences, University of Arkansas, Fayetteville, ArkanSAS 72701

Current address: Department of Mathematics, University of Maryland, College Park, Maryland 20742

E-mail address: shklover@wam.umd.edu 